Open Access

\title{
Water-Based Lubrication Preventing Side Grinding Demonstration in Medium-High Water Cut Wells
}

\author{
Lixia Fu ${ }^{*, 1}$, Yarong $\mathrm{Fu}^{1}$, Jun $\mathrm{Hao}^{1}$, Yunbo Shen ${ }^{2}$ and Qian $\mathrm{Fu}^{3}$
}

${ }^{1}$ Huabei Oilfield Company, Petrochina, Renqiu, Hebei 062552, China; ${ }^{2}$ Changqing Oilfield Com-
pany, Petrochina, Yulin, Shanxi 718500, China; ${ }^{3}$ Changcheng College, China University of Geosciences,
Baoding, Hebei 071000, China

\begin{abstract}
In order to extend the pump inspection cycle of the seriously-eccentric-wear well, FU-4 water-based lubricant, which can lubricate the boundary and form chemical reaction film and chemical adsorption film on the tubing and pump sucker rods surface, is synthesized by using water-based lubrication theory for reference which has been widely applied in the fields of cutting, grinding and others fields. The chemical reaction film and chemical adsorption film formed by the response activity of phosphorus, nitrogen and fatty acid molecules in the system for the carrier function have good abrasion resistance and anti-friction performance as well as good biodegradability. Adding water-based lubricant of $0.025 \%$ to $0.05 \%$ in the wells routine maintenance may extend the pump inspection cycle of eccentric-wear side grinding wells. It provides referable technique for managing side grinding wells.
\end{abstract}

Keywords: Medium-high water cut, oil well, Water-based lubrication, Abrasion resistance, Anti-friction.

\section{INTRODUCTION}

Recently, with the rapid growth development of petroleum industry, more and more people pay attention to the problem of water cut, and the working environment between the sucker rod and tubing is worsening in SRP(Sucker Rod Pumping) wells, making the phenomenon of eccentric wear between tubing and rod increasingly serious [1].

LI Han-zhou [2] found that the well track is the most important factor to affect the well eccentric wear in directional inclined borehole well; DANG Yong-feng [3] pointed out that factors such as the inclination, string vibration, high water cut and submergence depth are relevant to the eccentric wear of rod and tubing; Fu-qiang's [4] measures to prevent eccentric wear such as anti-wear collar, anti-wear pair, feedback pump do not work effectively due to the lack of relevant theoretical studies.

LIU Qing'e [5] hold that two-way wear proof collar, blade centering device and lined tubing have good effect on the anti-migraine grinding. However, the inner diameter of supporting lined tubing inner diameter is $7 \mathrm{~mm}$ smaller compared with that of ordinary tubing of the same specifications, and the piston effect increases the downstream resistance of sucker rod, flow volatility increases, rod loses stability, vibration becomes severe, rod and tubing eccentric wear shifts continuously $[6,7]$.

*Address correspondence to this author at the Huabei Oilfield Company, Petrochina, Renqiu, Hebei 062552, China;

Tel: 0317-2724207; E-mail: fulx2006@126.com
Macro tribology holds that [8], the basic phenomena in the sliding friction between the sucker rod and tubing such as surface contact, adhesion, abrasion and so on originated from bulk deformation of materials under load, but ignored the interactions of molecules between interface.

It is found that water-based lubricant with boundary lubrication effect can form chemical reaction film and chemical adsorption film on the tubing and sucker rod surface, i.e, a fixed inorganic film and a flowable organic layer formed on the friction surface take the role of boundary lubrication. Applying $0.025 \%$ to $0.05 \%$ more on wells at lined tubing and supporting two-way anti-eccentric wear the collar will extend the pump inspection by more than double.

\section{SYNTHESIS OF WATER-BASED LUBRICATION FOR OIL WELLS}

Water-based lubricant is widely used in the field of cutting, grinding, drawing, rolling, hydraulic transmission, etc [9].Additives such as surfactant, anti-wear additives and anti-rust agents can effectively improve anti-wear and scratch resistance performance of material under high temperature and high load [10].

Water-based lubricant used in well anti-eccentric-wear partially contains organic boron and organic boron-nitrogencontaining derivatives. Such anti-wear agent can form adsorption film in the friction surface under mild conditions, while boron and nitrogen decomposed under harsh conditions can penetrate into the surface and improve the wear resistance of it.

The percentages of weights taken by different components of water-based lubricant used for anti-eccentric wear are as follows [11]: polyoxyethylene glycerol boric 
acid ester stearic acid ester: 3.5\% 5.0\%; fatty acid imidazoline boric acid ester:3\% 5.5\%; amphoteric surfactant SS-Z: $\quad 4.5 \% \sim 7.0 \%$; fluorocarbon surfactant FN2:0.005\% 0.01\%; vitaminC:0.1\% 0.25\%; polyoxyethyle- ne alkyl phenol ether sodium salt: $11.5 \%$ 14.5\%; water for others. In the practical application, the percentages of the components need to make appropriate adjustments with the deference of well fluid component, eccentric wear degree, oil temperature and other different well conditions.

\section{Production Method}

Firstly, add the polyoxyethylene glycerol boric acid ester stearic acid ester, fatty acid imidazoline boric acid ester and amphoteric surfactant into enamel reactor prorata, and warm it slowly to $55 \sim 60^{\circ} \mathrm{C}$.

Secondly, add $5 \%$ of needed amount of water while stirring and then keep stirring for 30 minutes.

Thirdly, add the following items in sequence by a certain percentage: vitamin $\mathrm{C}$, fluorocarbon surfactant, FN-3 in proportion, while stirring; and then add polyoxyethylene alkyl phenol ether sodium salt in proportion, and keep stirring for 30 minutes; and then add the rest of the water and keep stirring for 30 minutes.

Finally, stop heating and keep stirring until it is cooled to normal temperature, then water-based lubricant FU-4 for well anti eccentric wear is achieved.

\section{PERFORMANCE EVALUATION OF WATER-BASED LUBRICANT IN OIL WELLS}

\section{The Film-Forming Properties}

Film-forming properties of the FU-4 solution are tested with NGY-6 nanometer scale film thickness gauge instrument, which measures lubricating film thickness of contact area with relatively light intensity [12].

Film-forming properties of water and 1\%, 5\%, 10\% FU-4 water-based lubricant are tested respectively, (Conditions: test load $20 \mathrm{~N}$ Hertz contact pressure: $50 \mathrm{MPa}$; temperature: $25 \pm 1{ }^{\circ} \mathrm{C}$ ). The results show that film-forming ability of water is week and it is difficult to form an effective lubricating film. By contrast, FU-4 solution can form the film thickness up to about $35 \mathrm{~nm}$ with concentration of $10 \%$, $23 \mathrm{~nm}$ with concentration of $1 \%$. However, when the concentration exceeds $5 \%$, its effect on the film-forming ability will become minimal.

\section{Friction and Wear Performance Test}

Anti-wear and extreme pressure performance test is taken for FU-4 aqueous solution under high load with MS-800 four-ball wear tester. Steel ball for test is the special GCr15 standard ball with diameter of $\Phi 12.7 \mathrm{~mm}$, made by Shanghai steel ball manufacture. The friction coefficient and ball wear scar of under different concentrations (see Table 1) of FU-4 water-based lubricant were tested by a 10-minite grinding test with static load $519 \mathrm{~N}$ and by the speed of $1438 \mathrm{r} / \mathrm{min}$.

As shown in Table 1, FU-4 water-based lubricant effectively improved the anti-wear and anti-fricction properties of material, for example, $0.5 \%$ content of the water-based lubricant could reduce the friction coefficient by $88.23 \%$ and wear scar diameter by $82.79 \%$, which indicates that the FU-4 water-based lubricant has good anti-wear property and anti-friction property. With content of waterbased lubricant increasing, the anti-friction property increases, the coefficient of friction reduces, wear scar diameter becomes smaller, which indicates that the anti-wear property increase.

Table 1. Friction and Wear Performance Test of FU-4 with Different Concentrations

\begin{tabular}{|c|c|c|}
\hline $\begin{array}{c}\text { Concentration of FU- } \\
\mathbf{4 ( \% )}\end{array}$ & $\begin{array}{c}\text { Friction } \\
\text { coefficient }\end{array}$ & $\begin{array}{c}\text { Wear scar diameter } \\
\mathbf{( m m})\end{array}$ \\
\hline \hline 0 & 1.02 & 2.15 \\
\hline 0.5 & 0.12 & 0.37 \\
\hline 1 & 0.09 & 0.32 \\
\hline 2 & 0.09 & 0.34 \\
\hline 3 & 0.10 & 0.33 \\
\hline 4 & 0.08 & 0.33 \\
\hline 5 & 0.07 & 0.30 \\
\hline 10 & 0.07 & 0.30 \\
\hline
\end{tabular}

Co-relation between the friction coefficient and the ball wear scar diameter for the FU-4 water-based lubricant of the same concentration under different loads is shown in Table 2. As it can be seen from the table, anti-wear and antifriction properties of $0.5 \%$ water-based lubricant changes little with the load increasing.

Table 2. Friction and Wear Performance Test of FU-4 with Different Loads

\begin{tabular}{|c|c|c|}
\hline Load (N) & Friction coefficient & Wear scar diameter (mm) \\
\hline \hline 519 & 0.12 & 0.37 \\
\hline 590 & 0.12 & 0.36 \\
\hline 649 & 0.11 & 0.37 \\
\hline 670 & 0.12 & 0.37 \\
\hline 745 & 0.13 & 0.38 \\
\hline
\end{tabular}

As Phosphorus and nitrogen in long-chain fatty acids in FU-4 water-based lubricant can be strongly adsorbed on the surface of the ball, boron and nitrogen, nitrogen can react chemically with friction surface, and generate high-strength friction chemical reaction film more easily, thus reducing the friction loss. Even under boundary lubrication conditions, the long-chain fatty acids can be quickly adsorbed on the friction surface and form a lubricating film, therefore the chemical reaction film will become more solid. Owing to the small atomic radius and high electronegativity of Nitrogen atom, it is easy to form hydrogen bonds, strengthen lateral gravity and increase the thickness of membrane during molecular friction, which, all contribute to enhance the strength of the surface film.

This experiment did not consider the effect of sand production in oil wells. On this issue we will further study in the next work. 


\section{Anti-rust Properties}

The anti-rust property of the FU-4 water-based lubricant with concentration of $0.25 \%, 0.5 \%, 1 \%, 2 \%, 3 \%, 4 \%, 5 \%$ and $10 \%$, in $35 \pm 1{ }^{\circ} \mathrm{C}$ thermostated container is studied [13]. Anti-rust performance increases with concentrations, when the concentration is $0.5 \%$, first order gray cast iron does not rust in a humid box, and be in accordance with GB6144-85 monolithic rust-proof standard.

\section{Biodegradation Capability}

CECL-33-A-93 method [14] determines the degradation rate of FU-4 water-based lubricant is $87.2 \%$, which indicates its good biodegradability.

\section{FIELD APPLICATION}

In the first application on wells, a $30 \mathrm{~m}^{3} 0.5 \% \sim 1 \%$ FU-4 water-based lubricant solution was injected from the annular space between the tubing and the tubing casing annulus by the tank truck, then take daily maintenance with $0.025 \%$ $\sim 0.05 \%$, after application in 30 more oil wells, the average pump inspection cyclecheck period is extend by more than doubled in the absence of other measures.

Typical well case:Well X-15,the'S'profile inclined well, daily fluid production rate is $12 \mathrm{~m}^{3}$, daily oil production rate is $\mathbf{1 . 5 \mathbf { m } ^ { 3 }}$, water cut is $87.5 \%$, the pump inspection cycle is only 90 days without any anti-eccentric-wear measure, the average pump inspection cycle of the oil wells used the lined tubing supporting with two-way preventing side wear collar is 154 days, and when adding $0.025 \% \sim 0.05 \%$ water-based lubricant in oil wells for routine maintenance, pump inspection cycle is up to 352 days.

\section{CONCLUSIONS}

The adsorption film and friction chemical reaction film formed in FU-4 water-based lubricant with high reaction activity of phosphorus, nitrogen and the fatty acid molecules carrier have excellent anti-friction performance.

During the period of wells routine maintenance, adding $0.025 \%$ to $0.05 \%$ water-based lubricant may extend the pump inspection cycle of the eccentric-wear wells. A referred technique for managing eccentric-wear wells is provided.

\section{CONFLICT OF INTEREST}

The authors confirm that this article content has no conflicts of interest.

\section{ACKNOWLEDGEMENTS}

Declared none.

\section{REFERENCES}

[1] J. Ying-Xiong, C. Fu-hua, Y. Rong-hong, W. Han-ping, J. Youhua, and Q. Yong, "On prevention of deviation from grinder in lubricating pumping pipes and rods with crude oil", Journal of Jianghan Petroleum University of Staff and Workers, vol. 21, no. 4, pp. 90-93, 2008.

[2] L. Han-zhou, Y. Hai-bin, M. Jian-jie, X. Guichun, and N. Houwen, "Relationship between well track and sucker rods eccentric wear", Drilling \& Production Technology, vol. 32, no. 3, pp. 81-82, 2009

[3] D. Yong-Feng, X. Guang-Jie, and S. Li-Hua, "Sucker rods eccentric wear and prevent measure study in shuanghe field", Journal of Oil and Gas Technology, vol. 31, no. 5 : pp. 396-398, 2009.

[4] F. Qiang, F. Lu-chang, and Z. Hong-bin, "Research and application of treatment process for lining tubing eccentric wear", Oil Field Equipment, vol. 37, no. 1 : pp. 84-87, 2008.

[5] L. Qing'e, T. Qing, and S. Fushan, "Selection and application of side wearing resistant tools for rods and pipes of rod pumped wells", Oil Drilling \& Production Technology, vol. 31, no. 2, pp. 111-114, 2009.

[6] W. Hai-wen, Z. Lei, Y. Feng, Z. Hui, H. Xiao-meng, and W. Wei-zhang, "Study on HDPE lined tubing in rod pumped wells", Oil Field Equipment, vol. 37, no. 2, pp. 74-77, 2008.

[7] F. Ya-rong, "Study and application of flushing fluid for oil wells with lined tubing", Oil Drilling \& Production Technology, vol. 33 , no. 5, pp. 110-112, 2011.

[8] G. Mang-lai, N. Shi-chun, and Z. Si-wei, "Effect of adhesion on nano-frictional properties of molecular deposition films", China Surface Engineering, vol. 16, no. 4, pp. 12-14, 2003.

[9] Z. Jie, W. Cheng-biao, G. Zhi-yong, W. Dan-ping, and L. Jiajun, "Effects of several additives on the properties of water-based lubricants", China Surface Engineering, vol. 20, no. 3, pp. 26-29, 2007.

[10] L. Xi-mei, and L. Xin-min, "The theory and application of waterbased lubricating additives", Synthetic Lubricants, vol. 28, no. 1, pp. 9-13, 2001.

[11] F. Ya-rong, W. Ze-mei, and J. Ying, "A anti-eccentric-wear lubrication in oil wells", China 201010146823, August 18, 2010.

[12] L. Jun-ming, Z. Chen-hui, Z. Chao-hui, W. Yan, and L. Si-si, "Lubricating Properties of the aqueous solution of castor oil polyoxyethylene ether", Tribology, vol. 31, no. 3, pp. 240-245, 2011.

[13] J. Hai-zhen, T. De-hua, W. Bin, H. Shu-gang, X. Juan, and Q. Lin-ling, "Synthesis and behavior of friction and wear of noleoylglutamic acid as water-based lubricant additive", Tribology, vol. 26, no. 1, pp. 45-48, 2006.

[14] X. Yun, F. Jian-hua, and W. Jiu, "The synthesis and tribological behaviors of phosphor-nitrogen modified fatty acid water base lubricant additive", Acta Petrolei Sinica (Petroleum Processing Section), vol. 25, no. S1, pp. 93-96, 2009.

Received: June 11, 2012

Revised: December 17, 2012

Accepted: December 18, 2012

(C) Fu et al.; Licensee Bentham Open.

This is an open access article licensed under the terms of the Creative Commons Attribution Non-Commercial License (http://creativecommons.org/licenses/by-nc/3.0/) which permits unrestricted, non-commercial use, distribution and reproduction in any medium, provided the work is properly cited. 